RM - $74-9$

international institute for RESEARCH MEMORANDUM

\title{
MINIMAL CONTROL FIELDS AND POLE-SHIFTING BY LINEAR FEEDBACK
}

J. Casti

April 1974 

Research Memoranda are informal publications relating to ongoing or projected areas of research at IIASA. The views expressed are those of the author, and do not necessarily reflect those of IIASA. 

Minimal Control Fields and

Pole-Shifting by Linear Feedback

J. Casti*

I. Introduction

From a practical point of view, the first consideration in the design of a controller for a physical system is for the resultant controlled system to he, in some sense, stable. The precise sense in which the stability is to be understood varies with the system requirements, but an often used criterion is that initial perturbations away from some desired equilibrium approach zero with increasing time, so-called asymptotic stability. Such considerations are well known in the engineering literature [1-2] and are being applied in many areas of contemporary interest such as ecology [3], urban systems [4], and economics [5].

The primary means for realizing the stability of a controlled system is by feedback. Some part of the output of the system is measured and compared with a desired level of system performance. The deviation of the actual behavior from the ideal is then used to generate an appropriate controlling input. This simple feedback idea has been used with great success in engineering systems for several decades and, as we have noted, is now explicitly finding its way into research

\footnotetext{
*International Institute for Applied Systems Analysis,
} Laxenburg, Austria and University of Arizona, Tucson, Arizona, U.S.A. 
other classes of systems although, in an implicit way, it has also been used in these areas for years since virtually all decision making involves such feedback notions.

Operationally, one of the central questions in the design of a stabilizing feedback control mechanism is: what measurements are necessary? It's quite possible, particularly in social and economic systems, that either all components of the state of the system may not be directly measurable or we may, at some expense measure all components of the state and wish to know the minimal number necessary in order to generate a stabilizing feedback law. The situation in which not all components are measurable has been treated in the literature by the introduction of a so-called "observer" [6]. It can be shown that, asymptotically, the fictitious system with an observer behaves in the same manner as the system with complete measurement capability. Technically, this result disposes of the question of incomplete observations. However, practically speaking such an approach has its drawbacks since in any real system the construction of an "observer" is not without cost in money, weight, reliability, etc. Thus, it is of some practical interest to ask what may be done in the way of stabilizing a system without introducing an observer or, in other words, what measurements are necessary to generate a stabilizing feedback control law.

In this note, we will examine the question of minimal control fields for constant linear systems. A complete solution to the problem, even for systems with a single input, seems 
extremely complicated and, at present, out of reach. The main difficulty stems from the fact that the solution is not coordinate-free and, consequently, the usual "canonical form" tricks of linear algebra seem to be of little use in arriving at a complete solution. However, results relating the minimal field problem to the question of assignment of characteristic values have been obtained and are reported here along with some possible approaches to the reneral problem and examples.

\section{Problem Statement}

To begin with, we consider the single-input constant linear system

$$
\dot{x}=F x+g u(t), \quad x(0)=c,
$$

where $F$ and $g$ are $n \times n$ and $n \times l$ matrices over the real numbers $R$, respectively, and $u(t)$ is a piecewise continuous scalar control law defined for all $t \geq 0$. We shall later consider the multi-input case when $G$ is a rectangular matrix of size $n \times m, 1<m \leq n$.

For our subsequent development, it is critical to assume that $\sum$ is completely controllable, i.e. the controllability matrix

$$
c=\left[g|F g| F^{2} g|\cdots| F^{n-1} g\right]
$$

has rank $n$ (is non-singular).

To avoid degeneracy of the problem, let us assume that $F$ has at least one characteristic value with non-negative real part and that $c \neq 0$. Thus, the feasible control $u \equiv 0$ will 
not stabilize $\sum$. Furthermore, we shall now restrict the admissible control actions $u$ to be of linear feedback type, i.e. $u(t)=k^{\prime} x(t)$, where $k$ is an $n x 1$ constant vector over $P$ whose components are to be chosen so that the controlled system

$$
\dot{x}=\left(F+\pi k^{\prime}\right) x
$$

is asymptotically stable, i.e. the characteristic roots of $F+g K^{\prime}$ lie in the left half-plane. Under complete controllability of $[$, the "pole-shifting" theorem of linear systems theory insures that, given any symmetric set of $n$ complex numbers, it is possible to find a unique $k$ such that $F+g k^{\prime}$ has the given set of numbers as its characteristic values. This establishes the existence of a stabilizing control law. We may now state the

Basic Problem of Minimal Control Fields (BP):

Determine a vector $k$ such that

(i) $F+g k^{\prime}$ is a stability matrix,

(ii) $k$ has the maximum number of zero components.

The minimal field condition (ii) indicates that if $k$ is a stabilizing law and $k_{i}=0$, then component $x_{i}$ of the state vector does not appear in the feedback law $g k^{\prime}$, i.e. it is not necessary to measure $x_{i}$ to stabilize $\sum$ by feedback. Results relating to the $B P$ were given by casti and Letov in [7]. In [7] the system $\sum$ was considered without the assumption 
of linear feedback and upper bounds were obtained on the number of components of $x$ appearing in a minimal field. However, the technique used does not readily extend to obtaining the exact dimension of a minimal field.

Since the BP seems out of reach at the moment, we consider the following modified version:

The Prescribed Poles and Minimal Field Problem (PP):

Given a symmetric set of $n$ complex numbers which are to be the characteristic values of the controlled system $\Sigma^{\prime}$, determine those components of $x$ which may be omitted from the measurements which generate the linear control law.

In the next section, we shall give a complete solution to $P P$ including a test for determining whether or not a given component of $x$ may be omitted in a control law achieving the desired distribution of characteristic values for $\Sigma^{\prime}$. From an operational point of view, PP may be even more important than $B P$ since it enables us to prescribe in advance the rapidity with which $\mathrm{x}$ will approach the origin at the expense, perhaps, of an increased size of the minimal field.

\section{The Solution of PP}

To give a reasonably concise solution to $P P$, it is convenient to reduce $\{$ to control canonical form, i.e. introduce a basis in the state space so that $F$ and $g$ assume the forms 


$$
\begin{aligned}
& \underset{F}{T} \hat{F}= {\left[\begin{array}{ccccc}
0 & 1 & 0 & \cdots & 0 \\
0 & 0 & & \cdots & 0 \\
\vdots & & & \cdots & \\
0 & 0 & 0 & \cdots & 1 \\
-\alpha_{n} & -\alpha_{n-1} & -\alpha_{n-2} & \cdots & -\alpha_{1}
\end{array}\right], } \\
& \underset{g \rightarrow \hat{g}}{T}=\left(\begin{array}{c}
0 \\
0 \\
\vdots \\
0 \\
1
\end{array}\right) .
\end{aligned}
$$

where the $a^{\prime} s$ are the coefficients in the characteristic polynomial $x(z)$ of $F$,

$$
\underset{F}{x}(z)=z^{n}+\alpha_{1} z^{n-1}+\alpha_{2} z^{n-2}+\cdots+\alpha_{n} .
$$

The transformation $T$ which effects this change is given explicitly by

$$
T=\left[\hat{g}|\hat{F} \hat{g}| \hat{F}^{2} \hat{g}|\cdots| \hat{F}^{n-1} \hat{g}\right]\left[g|F g| \cdots \mid F^{n-1} g\right]^{-1} .
$$

This form clearly points out the necessity and sufficiency of complete controllability for $[$ in our results. In the canonical coordinate system, we must consider the system

$$
z=\left(\hat{F}+\hat{g} \hat{k}^{\prime}\right) z, \quad\left(\Sigma_{\text {can }}\right)
$$

where $\hat{k}^{\prime}=\mathrm{k}^{\prime} \mathrm{T}^{-1}$. The systems $\sum_{\text {can }}$ and $\sum^{\prime}$ will have the same characteristic values since $T$ is a similarity transformation. 
Our main result is

Theorem 1. Let $\Lambda=\left\{\lambda_{1}, \lambda_{2}, \ldots, \lambda_{n}\right\}$ be a given symmetric set of complex numbers and let $x_{\hat{F}}+\hat{g} \hat{k}(\underline{z})-\prod_{i=1}^{n}\left(z-\lambda_{i}\right)=z_{n}+\beta_{1} z^{n-1}+\cdots \beta_{1}$ be the desired characteristic polynomial of the controlled system. Then the component $x_{i}$ will not appear in the feedback law k generating the system $\sum^{\prime}$ if, and only if, $\left(k, T^{(i)}\right)=0$, where $T^{(i)}$ is the $i^{\text {th }}$ column of the matrix $T$, $i=1,2, \ldots, n, i . e . \hat{k}$ must be orthogonal to the $i^{\text {th }}$ column of $T$.

Proof. The particular forms of $\hat{F}$ and $\hat{g}$ show that $\hat{\mathrm{F}}+\hat{\mathrm{g}} \hat{k}^{\prime}=\left[\begin{array}{ccccc}0 & 1 & 0 & \cdots & 0 \\ 0 & 0 & 1 & \cdots & 0 \\ 0 & 0 & 0 & \cdots & 1 \\ -\alpha_{n+\hat{k}_{1}} & -\alpha_{n-1+\hat{k}_{2}} & -\alpha_{n-2+\hat{k}_{3}} & \cdots & -\alpha_{1}+\hat{k}_{n}\end{array}\right]$. Thus, if the characteristic polynomial of $\hat{F}+\hat{g} \hat{k}$ ' is to be $x \hat{F}+\hat{g} \hat{k}^{\prime}(z)=z^{n}+\sum_{i=1}^{n} B_{i} z^{n-1}$, we must choose $k_{i}=\alpha_{n-i+1}-\beta_{n-i+1}$, $i=1,2, \ldots, n$. This uniquely determines $k$. Transforming back to the original coordinate system, we have $k^{\prime}=k^{\prime} T$. Thus, $k_{i}=0$ if, and only if, $\hat{k}^{\prime} T^{(i)}=0$, i.e. $k$ is orthogonal to the $i^{\text {th }}$ column of $T$. Hence component $x_{i}$ will appear in the feedback law generating $\sum^{\prime}$ unless $\hat{k}^{\prime T}(i)=0$.

The following re-statement of the theorem gives a simple test for deciding whether or not certain components of $x$ may be omitted in a law generating a system having $\Lambda$ as its characteristic values: 
Corollary. Let $\mathrm{W}$ be the subspace of $\mathrm{R}^{\mathrm{n}}$ generated by the vectors $T^{\left(i_{1}\right)}, T{ }^{\left(i_{2}\right)}, \ldots, T^{\left(i_{m}\right)}$ and let $\hat{k}$ be the control law generating a $\sum^{\prime}$ having $\Lambda$ as its set of characteristic roots. Then components $x_{i_{1}}, x_{i_{2}}, \ldots, x_{i_{m}}$ will not appear in the control law $k$ if, and only if, kew, the orthogonal complement of $w$.

\section{Some Examples}

In this section we present a few simple examples illustrating the use of the results given above.

Example 1. Assume that $F$ and $g$ are given in the control canonical form

$$
F=\left[\begin{array}{llll}
0 & 1 & 0 & 0 \\
0 & 0 & 1 & 0 \\
0 & 0 & 0 & 1 \\
0 & 4 & 4 & 1
\end{array}\right], \quad E=\left(\begin{array}{l}
0 \\
0 \\
0 \\
1
\end{array}\right) .
$$

Thus, the characteristic polynomial of $F$ is

$$
\begin{aligned}
\chi_{F}(z) & =z^{4}-z^{3}-4 z^{2}+4 z \\
& =(z-1)(z+2)(z)(z-2) .
\end{aligned}
$$

This system has the three unstable roots 1,2,0. Assume we wish the controlled system to have the characteristic values -2 , $-1,-1,-1$. Hence

$$
\begin{aligned}
X_{F-g k}(z) & =(z+1)^{3}(z+2) \\
& =z^{4}+5 z^{3}+9 z^{2}+7 z+2 .
\end{aligned}
$$


Comparing $\chi_{F}$ and $\chi_{F-g k}$, we see that

$$
k^{\prime}=\left(\begin{array}{llll}
-2 & -3 & -13-6
\end{array}\right)
$$

Since the basic transformation matrix for this example is $T=I$, $k^{\prime}\left(=\hat{k}^{\prime}\right)$ is not orthogonal to any column of $T$. Hence, all components of the state will appear in the feedback law which produces $X_{F-F k} \cdot \cdot$

Now assume that we desire the characteristic roots to be $-1,-1,-1,-1$. Then

$$
\begin{aligned}
x_{F-g K^{\prime}}(z) & =(z+1)^{4} \\
& =z^{4}+4 z^{3}+6 z^{2}+4 z+1 .
\end{aligned}
$$

Hence, $k^{\prime}=(-1,0,-10,-5)$ and we see that $k^{1} \perp T^{(2)}, i . e . c o m-$ ponent $x_{2}$ does not appear in the control law.

Example 2. Let $F$ and $g$ be given by

$$
F=\left[\begin{array}{lll}
0 & 0 & 0 \\
1 & 0 & 0 \\
0 & 1 & 1
\end{array}\right], \quad g=\left(\begin{array}{l}
1 \\
0 \\
0
\end{array}\right)
$$

It's easily verified that $[$ is completely controllable $(C=I)$ and the control canonical form is

$$
\hat{F}=\left[\begin{array}{lll}
0 & 1 & 0 \\
0 & 0 & 1 \\
0 & 0 & 1
\end{array}\right], \quad \hat{g}=\left(\begin{array}{l}
0 \\
0 \\
1
\end{array}\right) .
$$

The matrix of the basis transformation is

$$
T=\left[\begin{array}{lll}
0 & 0 & 1 \\
0 & 1 & 1 \\
1 & 1 & 1
\end{array}\right]
$$


The original system has the characteristic values $1,0,0$. We assert that it is not possible to stabilize this system by any linear feedback law which does not measure all state components. For example, to eliminate $x_{3}$, by the orthogonality condition we must have

$$
k_{1}+k_{2}+k_{3}=0 \text {, }
$$

where $k^{\prime}=\left(k_{1} k_{2} k_{3}\right) \cdot$ But

$$
x_{F}+\hat{g} \hat{k},(z)=z^{3}-\left(k_{3}+1\right) z^{2}-k_{2} z-k_{1}
$$

and the necessary condition for a stability polynomial is that all coefficients be positive. Hence

$$
k_{1}<0, \quad k_{2}<0, \quad k_{3}<-1 \text {, }
$$

which is inconsistent with the orthogonality requirement. A similar argument holds for the elimination of $x_{1}$ or $x_{2}$. Thus, the only way this system can be stabilized is if all components of the state are fed back.

\section{Multi-input Systems}

Now we briefly consider the situation when $\sum$ has more than one input, i.e.

$$
\dot{\mathrm{x}}=\mathrm{Fx}+\mathrm{Gu} \text {, }
$$

where $G$ is an $n \times m$ constant matrix with $1<m \leq n$. 
The linear feedback law is now

$$
\mathrm{u}=\mathrm{Kx} \text {, }
$$

where $K$ is an $m \times n$ matrix and the system $L^{\prime}$ becomes

$$
x^{\prime}=(F+G K) x
$$

The control canonical form of $[$ is somewhat more complex than in the single-input case since now changes of bases in both the state and control spaces are required. The details of these transformations are discussed in [10]. The final result is, that for $(F, G)$ completely controllable, it is possible through basis changes to bring $F$ and $G$ to the forms

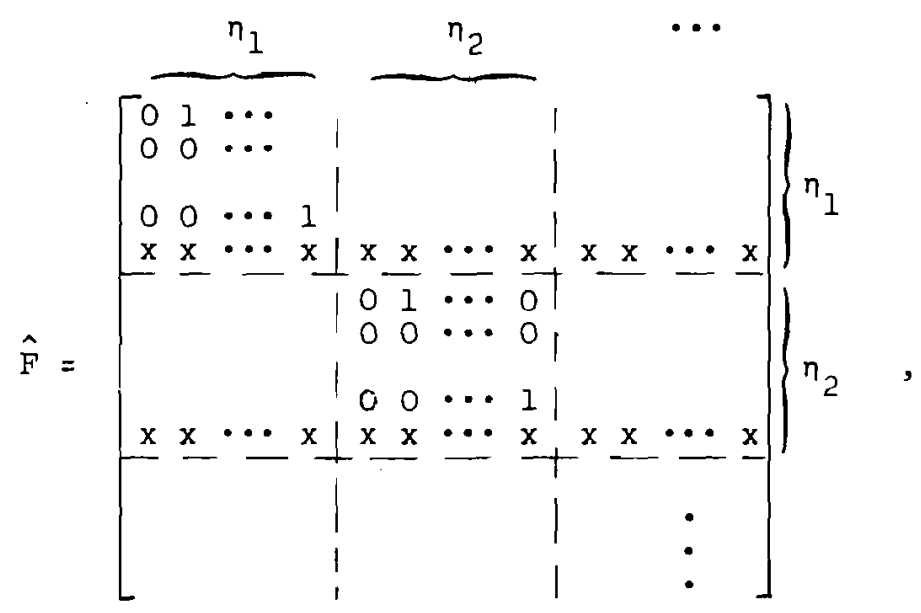




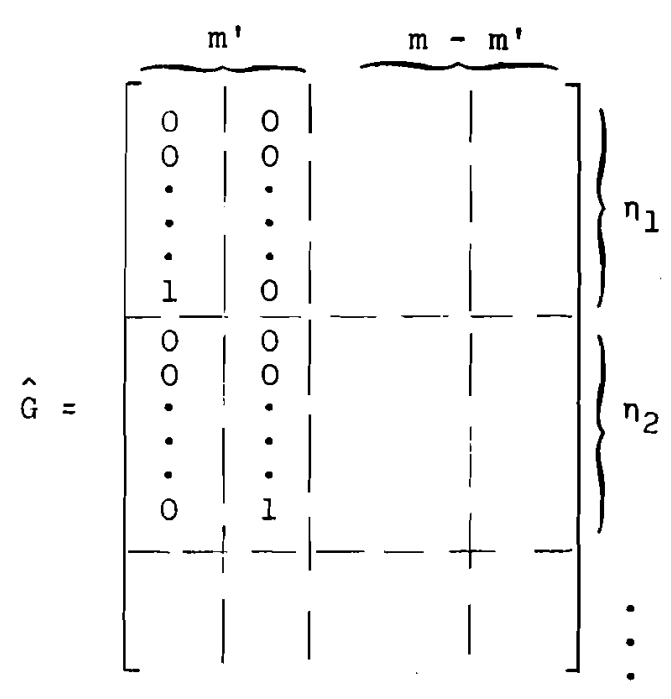

Here the numbers $n_{l}, \ldots, n_{k} \sum_{i=1}^{k} n_{i=n}$, are the Kronecker indices of the pencil of matrices generated by $F$ and $G$. The number $m^{\prime}$ refers to the number of columns of $G$ which are used in constructing, the new basis by the prescription given in [10]. The quantities denoted by " $x$ " in $\hat{F}$ are also determined by the precise form of the basis change described in [10].

Let $\hat{K}$ be the feedback law which corresponds to the desired placement of the characteristic values of $\sum^{\prime}$ and let $T$ be the matrix of the basis transformation in the state space. Then we have

Theorem 2. Component $x_{i}$ of $\sum$ may be omitted from the feedback law generating $\sum^{\prime}$ if, and only if, $\mathrm{T}^{(i)}$ is orthogonal to each row of $\hat{k}$, $i=1,2, \ldots, n$. 
Proof. Follows immediately from the fact that

$$
K=\hat{K} T \text {. }
$$

For definiteness, the matrix $T$ is given by

$$
\begin{aligned}
T= & {\left[\hat{G}|\hat{F G}| \cdots \mid \hat{F}^{n-r_{G}}\right]\left[\hat{G}|\hat{F G}| \cdots \mid \hat{F}^{n-r_{G}}\right], } \\
& \left\{\left[G|F G| \cdots \mid F^{n-r} G\right]\left[G|F G| \cdots \mid F^{n-r_{G}}\right]^{\prime}\right\}-1,
\end{aligned}
$$

where $r=\operatorname{rank} G$. For a proof of this representation, see [8].

\section{Discussion}

The foregoing results raise several interesting questions which merit further investigation, in addition to the BP. Among these are:

i) Since the minimal control field is coordinate dependent, what is the "optimal" coordinate system? Here "optimal" is understood in the sense of smallest number of state components appearing in the feedback law;

ii) how can the above results be extended to non-linear and discrete-time systems? For example, the Poincare-Lyapunov Theorem [9] insures that Theorems 1 and 2 are still valid for systems of the form

$$
\text { t }=F x+G u+f(x), \quad x(0)=c \text {, }
$$

when $\frac{|| f(x)||}{|| x||}+0$ as ||$x||+0$ and $\| c||$ is sufficiently small; 
iii) if the input matrix $G$ is at our disposal, in what manner should it be selected to make the size of the minimal control field as small as possible? In [7], it was shown that for a certain non-linear feedback law, the minimal field had dimension one if $G$ was arbitrary. 


\section{References}

[1] Hahn, W. Stability of Motion. New York, SpingerVerlag, 1967.

[2] LaSalle, J. and Lefschetz, S. Stability by Lyapunov's Direct Method with Applications. New York, Academic Press, 1961.

[3] Holling, C.S. "Resilience and Stability of Ecological Systems," Ann. Review Ecol. Systems, 4 (1973).

[4] Domanski, R. "Structure, Law of Motion, and Optimal Path of Growth of Complex Urban Systems," Economic Geography, 49 (1973), 37-46.

[5] Intriligator, I. Mathematical Optimization and Economic Theory. Englewood Cliffs, N.J., Prentice-Hall Co., 1971.

[6] Luenberger, D. "Observing the State of a Linear System," IEEE Trans. Military Electronics, MIL-8 (1964), $74-80$

[7] Casti, J. and Letov, A. "Minimal Control Fields," J. Math. Analy. Applic., 43 (1973), 15-25.

[8] Kalman, R., Falb, P., and Arbib, M. Topics in Mathematical System Theory. New York, McGraw-Hill Co., 1969.

[9] Bellman, R. Stability Theory of Differential Equations. New York, McGraw-Hill, 1953.

[10] Kalman, R. "Kronecker Invariants and Feedback," in Ordinary Differential Equations: Proceedings of 1971 NRL Conference, I. Weiss, ed. New York, Academic Press, 1973. 